\title{
Depiction of Intuitionistic Fuzzy Soft Linear Spaces
}

\section{Siva Naga Malleswari, V. Amarendra Babu}

\begin{abstract}
We define Intuitionistic fuzzy soft linear spaces (IFSLS) and its properties and characteristics are studied with examples. We propose thedefinition "Cartesian product of Intuitionistic fuzzy soft linear spaces" and these are illustrated by some examples. Besides that, we define Intuitionistic soft subspaces and given examples.
\end{abstract}

Keywords: Intuitionistic fuzzy soft linear space (IFSLS), Intuitionistic fuzzy soft subspace (IFSSS).

\section{INTRODUCTION}

Molodtsov [2] introduces the definition of soft set. K.Attansov [3][4] introduces the intuitionistic fuzzy set. The hypothesis of intuitionistic fuzzy soft set was proposed by Maji et al [6]. Moumita Chiney and S.K.Samanta describes the concept of Intuitionistic fuzzy vector spaces [5]. A.Sezgin Sezer, A.O.Atagin introduced the concept of soft vector spaces [1].

In this paper, we introduce intuitionistic fuzzy soft linear spaces (IFSLS) and some of its properties and characteristics are studied with examples. We propose the definition "Cartesian product of Intuitionistic fuzzy soft linear spaces" and these are illustrated by some examples. Besides that, we define Intuitionistic soft subspaces and given examples.

\section{PRELIMINARIES}

Definition 2.1: A continuous t-norm is defined by a binary operation $*:[0,1] X[0,1] \rightarrow[0,1]$ if $*$ satisfies the below mentioned properties

(i) $s * t=t * s$

(ii) $(s * t) * u=s *(t * u)$

(iii) $\quad *:[0,1] X[0,1] \rightarrow[0,1]$ is continuous

(iv) $s * 1=1 * s$

(v) $s * t \leq u * v i f s \leq u, t \leq v$ for all $s, t, u, v \in$

Some examples are $s * t=s t, s * t=\min \{s, t\}, s * t=$ $\max \{s+t, 1\}$.

Definition 2.2: A continuous t-co norm(s-norm) is defined by a binary operation $\Delta:[0,1] X[0,1] \rightarrow[0,1]$ if $\Delta$ satisfies the below mentioned properties

Revised Manuscript Received on December 15, 2019.

V. Siva Naga Malleswari, Assistant Professor, PVPSIT, A.P, India. Email: vsnm.maths@gmail.com

Dr. V. Amarendra Babu, Department of Mathematics, Acharya Nagarjuna University, Nagarjuna Nagar, India.

E-mail: amarendravelisela@ymail.com (i)

(ii) $(s \Delta t) \Delta u=s \Delta(t \Delta u)$

(iii)

(iv)

$$
\Delta:[0,1] X[0,1] \rightarrow[0,1] \text { is continuous }
$$
$s \Delta 0=0 \Delta s$ $s \Delta t \leq u \Delta v i f s \leq u, t \leq v$ for all $s, t, u, v \in$

Some examples $\quad \operatorname{are} s \Delta t=s+t-s t, s \Delta t=$ $\max \{s, t\}, s \Delta t=\min \{s+t, 1\}$.

If $s * s=s$ then $*$ is called an idempotent t-norm and if $s \Delta s=s$ then $\Delta$ is called an idempotent s-norm $\forall s \in[0,1]$.

Definition 2.3: Let $x$ be an element in $X$ which is the space of points (objects). An intuitionistic fuzzy set $\mathrm{A}$ in $\mathrm{X}$ is defined by $T_{A}: X \rightarrow[0,1]$, which is called a truth membership function $\operatorname{and} F_{A}: X \rightarrow[0,1]$, which is falsity membership function and $0 \leq T_{A}(x)+F_{A}(x) \leq 1$.

Definition 2.4: If $\mathrm{S}$ is a mapping defined by $\mathrm{S}: B \rightarrow P(U)$ then a pair $(\mathrm{S}, \mathrm{B})$ is known as a soft set over $U$ for $B \subseteq E$. Here $P(U)$ denotes the power set of $\mathrm{U}, \mathrm{U}$ denotes an initial universal set and the set of parameters is denoted by $\mathrm{E}$.

Definition 2.5: Let $\mathrm{F}$ be a mapping given by $F: S \rightarrow I F(U)$ then a pair $(\mathrm{F}, \mathrm{S})$ is known as an intuitionistic fuzzy soft set over $\mathrm{U}$ for $\mathrm{S} \subseteq E$. Here the initial universal set is denoted by $\mathrm{U}$ and $\mathrm{E}$ denotes a set of parameters.

If $F(s)$ is an Intuitionistic fuzzy set of $\mathrm{U}$ for every $s \in S$ then it is called an Intuitionistic fuzzy value set of parameters. Then $F(s)$ is an Intuitionistic fuzzy value set such that $F(s)=\left\{\left\langle x, \mu_{F(s)}(x), \lambda_{F(s)}(x)>/ x \in U\right\}\right.$ where $\mu_{F(s)}$ is a membership function and $\lambda_{F(s)}$ is a non membership function. Then intuitionistic fuzzy soft class is defined as set of all intuitionistic fuzzy soft sets over $U$ with parameters from $\mathrm{E}$ and is denoted by $\operatorname{IFF}(\mathrm{U}, \mathrm{E})$.

\section{Example 2.6:}

Consider $U=\left\{\mathrm{v}_{1}, \mathrm{v}_{2}, \mathrm{v}_{3}\right\}$ be a set of vehicles and $\mathrm{E}=$ $\left\{\mathrm{e}_{1}\right.$ (mileage), $\mathrm{e}_{2}$ (cheap), $\mathrm{e}_{3}$ (costly) $\}$ be a set of parameters w.r.t which the nature of vehicles are described. Let 


\section{Depiction of Intuitionistic Fuzzy Soft Linear Spaces}

$\mathrm{f}\left(\mathrm{e}_{1}\right)=\left\{\left\langle\mathrm{v}_{1}(0.5,0.3)\right\rangle,\left\langle\mathrm{v}_{2}(0.4,0.6)\right\rangle,\left\langle\mathrm{v}_{3}(0.6,0.3)\right\rangle\right\}$

$\mathrm{f}\left(\mathrm{e}_{2}\right)=\left\{\left\langle\mathrm{v}_{1}(0.6,0.3)\right\rangle,\left\langle\mathrm{v}_{2}(0.7,0.3)\right\rangle,\left\langle\mathrm{v}_{3}(0.8,0.2)\right\rangle\right\}$

$f\left(e_{3}\right)=\left\{\left\langle v_{1}(0.7,0.3)\right\rangle,\left\langle v_{2}(0.6,0.2)\right\rangle,\left\langle v_{3}(0.5,0.2)\right\rangle\right\}$

Then $\mathrm{I}=\left\{\left\langle\mathrm{e}, \quad \mathrm{f}_{\mathrm{I}}\left(\mathrm{e}_{1}\right)\right\rangle,\left\langle\mathrm{e}_{2}, \mathrm{f}_{\mathrm{I}}\left(\mathrm{e}_{2}\right)>,\left\langle\mathrm{e}_{3}, \mathrm{f}_{\mathrm{I}}\left(\mathrm{e}_{3}\right)\right\rangle\right\} \quad\right.$ is $\quad$ an intuitionistic fuzzy soft set over (U,E).

Definition2.7: The complement of IFSS I is denoted by $\mathrm{I}^{\mathrm{c}}$ and it is defined as

$\mathrm{I}^{\mathrm{c}}=\left\{\mathrm{e},<\mathrm{x}, \mathrm{F}_{\mathrm{I}}(\mathrm{e}), \mathrm{T}_{\mathrm{I}}(\mathrm{e})>/ \mathrm{x} \in U, e \in E\right\}$.

Definition 2.8: Let $I_{1}$ and $I_{2}$ be two IFS sets over (U, E).

Then $\mathrm{I}_{1}$ is said to be an intuitionistic fuzzy soft subset of $\mathrm{I}_{2}$ $\operatorname{ifT}_{f_{I}\left(e_{1)}\right.}(x) \leq T_{f_{I}\left(e_{2}\right)}(x), F_{f_{I}\left(e_{1}\right)}(x) \geq F_{f_{I}\left(e_{1)}\right.}(x) \forall \mathrm{e} \in E, x \in$ $U$.

Definition 2.9: Let $I_{1}$ and $I_{2}$ be two IFS sets over (U,E). The union is denoted by $I_{1} \cup I_{2}=I_{3}$ and it is defined as:

$\mathrm{I}_{3}=\left\{\mathrm{s},\left\langle\mathrm{x}, T_{f_{I_{3}}(s)}(x), F_{f_{I_{3}}(s)}(x)>/ \mathrm{x} \in U, s \in E\right\}\right.$

Where $T_{f_{I_{3}}(s)}(x)=T_{f_{I_{1}}(s)}(x) \Delta T_{f_{I_{2}}(s)}(x)$

$F_{f_{I_{3}}(s)}(x)=F_{f_{I_{1}}(s)}(x) * F_{f_{I_{2}}(s)}(x)$

Their intersection is defined by $\mathrm{I}_{1} \cap \mathrm{I}_{2}=\mathrm{I}_{4}$ and it is defined as $\mathrm{I}_{4}=\left\{\mathrm{s},\left\langle\mathrm{x}, T_{f_{I_{4}}(s)}(x), F_{f_{I_{4}}(s)}(x)>/ \mathrm{x} \in U, s \in E\right\}\right.$

Where $T_{f_{I_{4}}(s)}(x)=T_{f_{I_{1}}(s)}(x) * T_{f_{I_{2}}(s)}(x)$

$F_{f_{I_{4}}(s)}(x)=F_{f_{I_{1}}(s)}(x) \Delta F_{f_{I_{2}}(s)}(x)$

Definition 2.10: Let $I_{1}$ and $I_{2}$ be two IFSS over (U, E).Then 'and' operation is denoted by

$\mathrm{I}_{1} \wedge \mathrm{I}_{2}=\mathrm{I}_{5}$ and it is defined as

$\mathrm{I}_{5}=\left\{(\mathrm{s}, \mathrm{t}) /<\mathrm{x}, T_{f_{I_{5}}(s, t)}(x), F_{f_{I_{5}}(s, t)}(x)>/ \mathrm{x} \in U,(s, t) \in E X E\right\}$

Where $T_{f_{I_{5}}(s, t)}(x)=T_{f_{I_{1}}(s)}(x) \Delta T_{f_{I_{2}}(t)}(x)$

$F_{f_{I_{5}}(s, t)}(x)=F_{f_{I_{1}}(s)}(x) * F_{f_{I_{2}}(t)}(x)$

Definition 2.11: An IFS set I over (U,E) is said to be a null IFS set if $T_{f_{I}(e)}(x)=0, F_{f_{I}(e)}(x)=1 \quad \forall$ e $\in E, x \in U$ and isdenoted by $\emptyset_{I}$.

An IFS set I over (U, E) is said to be absolute IFS set if $T_{f_{I}(e)}(x)=1, F_{f_{I}(e)}(x)=0 \forall \mathrm{e} \in E, x \in U$ and is denoted by $I_{A}$.

\section{INTUITTIONISTIC FUZZY SOFT LINEAR SPACES}

Definition 3.1: An Intuitionistic fuzzy set $\mathrm{B}=\{<$ $\left.x, T_{B}(x), F_{B}(x)>x \in V\right\}$ on a vector space $\mathrm{V}(\mathrm{K})$ is called an intuitionistic fuzzy sub vector space $\mathrm{V}(\mathrm{K})$ if

(i) $\quad T_{B}(x+y) \geq T_{B}(x) * T_{B}(y)$

(ii) $\quad F_{B}(x+y) \leq F_{B}(x) \Delta F_{B}(y) \forall x, y \in V$ (iii) $\quad T_{B}(\lambda x) \geq T_{B}(x)$

(iv) $\quad F_{B}(\lambda x) \leq F_{B}(x) \forall x, y \in V, \lambda \in K$.

An intuitionistic fuzzy soft set $\mathrm{I}$ on $\mathrm{V}(\mathrm{K})$ is said to be an intuitionistic fuzzy soft vector space/ linear space (IFSLS) if $f_{I}(\mathrm{e})$ is a intuitionistic fuzzy sub vector space on $\mathrm{V}(\mathrm{K})$ for all e $\in E$.

Example3.2: If $E=\left\{\mathrm{e}_{1}, \mathrm{e}_{2}, \ldots . ., \mathrm{en}\right\}$ denotes the parametric set and $\mathrm{R}^{\mathrm{n}}(\mathrm{R})$ be the $\mathrm{n}$-dimensional Euclidean space. Let us define a mapping $f_{I}: E \rightarrow \operatorname{IFS}\left(R^{n}\right)$ for any $\mathrm{t} \in R^{n}$ as following:

$T_{f_{I}(e)}(t)=\left\{\begin{array}{c}\frac{1}{2}, \text { ifithcoordinateoftiszero } \\ 0, \text { otherwise }\end{array}\right.$

$F_{f_{I}(e)}(t)=\left\{\begin{array}{c}0, \text { if ithcoordinateoftiszero } \\ 1 / 10, \text { otherwise }\end{array}\right.$

If $a * b=\min \{a, b\}, a \Delta b=\max \{a, b\}$.

Then I forms an IFSS as well as IFSLS over $\mathrm{R}^{\mathrm{n}}(\mathrm{R})$ w.r.t parametric set E.

For convenience, we take an attempt for the parameter e and Euclidean space $\mathrm{R}^{2}(\mathrm{R})$.

Then the following four cases arise to choose $\mathrm{x}, \mathrm{y} \in R^{2}$.

Case I: If $\mathrm{x}=(0,4)$ and $\mathrm{y}=(0,2)$ then $\mathrm{x}+\mathrm{y}=(0,6)$

Case II: If $\mathrm{x}=(0,3)$ and $\mathrm{y}=(3,2)$ then $\mathrm{x}+\mathrm{y}=(3,5)$

Case III: If $\mathrm{x}=(1,2)$ and $\mathrm{y}=(5,1)$ then $\mathrm{x}+\mathrm{y}=(6,3)$

Case IV: If $\mathrm{x}=(5,1)$ and $\mathrm{y}=(-5,4)$ then $\mathrm{x}+\mathrm{y}=(0,5)$

From these four cases, the first and second set of conditions can be verified.

Example 3.3: Consider a real vector space $C=\{a+i b / a, b \in$ $R, \mathrm{i}=\sqrt{-1}\}$ and the parametric set $\mathrm{E}=\{\alpha, \beta, \gamma\}$. We divide the elements of $\mathrm{C}$ into four cases e.g:

(C1) $\{\mathrm{ib} / \mathrm{b} \in R-\{0\}\}$ when real part is zero

(C2) $\{\mathrm{a} / \mathrm{a} \in R-\{0\}\}$ when imaginary part is zero

(C3) $\{\mathrm{a}+\mathrm{ib} / \mathrm{a}, \mathrm{b} \in R-\{0\}\}$ when both parts are non zero

(C4) $\{0+\mathrm{i} 0\}$ the null vector

If $x \in C 1$ and $y \in C 2$ then $x+y \in C 3$. We write $C 1+C 2=C 3$.

We define IFSS I over $(\mathrm{C}, \mathrm{E})$ is given by $a * b=\max \{\mathrm{a}+\mathrm{b}-1$, $0\}, \mathrm{a} \Delta b=\{a+b, 1\}$.Then I forms an IFSLS over (C(R),E).

From these four cases, the first and second set of conditions can be verified.

Corollary 3.4: Let I be an IFSLS over $(\mathrm{V}(\mathrm{K}), \mathrm{E})$.Then for $\mathrm{x} \in V$

and 
$\lambda(\neq 0) \in K, T_{f_{I}(e)}(\lambda x)=T_{f_{I}(e)}(x), F_{f_{I}(e)}(\lambda x)=F_{f_{I}(e)}(x)$ hold.

Proof: $T_{f_{I}(e)}(x)=T_{f_{I}(e)}\left(\lambda^{-1}(\lambda x)\right) \geq T_{f_{I}(e)}(\lambda x)$

$F_{f_{I}(e)}(x)=F_{f_{I}(e)}\left(\lambda^{-1}(\lambda x)\right) \leq F_{f_{I}(e)}(\lambda x)$

Now from the second set of conditions in definition of IFSLS , the result follows.

\section{Proposition 3.5:}

Let $\mathrm{I}$ be an IFSLS over (V (K), E). Then for each $t \in \mathrm{V}$, following hold.

$$
\mathrm{T}_{\mathrm{f}_{\mathrm{I}}(\mathrm{e})}(-t)=\mathrm{T}_{\mathrm{f}_{\mathrm{I}}(\mathrm{e})}(t), \mathrm{F}_{\mathrm{f}_{\mathrm{I}}(\mathrm{e})}(-t)=\mathrm{F}_{\mathrm{f}_{\mathrm{I}}(\mathrm{e})}(t)
$$

$$
\mathrm{T}_{\mathrm{f}_{\mathrm{I}}(\mathrm{e})}(\theta) \geq \mathrm{T}_{\mathrm{f}_{\mathrm{I}}(\mathrm{e})}(t), \mathrm{F}_{\mathrm{f}_{\mathrm{I}}(\mathrm{e})}(\theta) \leq \mathrm{F}_{\mathrm{f}_{\mathrm{I}}(\mathrm{e})}(t)
$$

if $a * b=\min \{a, b\}, a \Delta b=\max \{a, b\}$ and $\theta$ is null vector of $\mathrm{V}$.

Proof :(i) For $\lambda=-1$, the result directly follows from above corollary.

(ii)For the null vector $\theta \in V$

$\mathrm{T}_{\mathrm{f}_{\mathrm{I}}(\mathrm{e})}(\theta)=\mathrm{T}_{\mathrm{f}_{\mathrm{I}}(\mathrm{e})}(t+(-t)) \geq$

$\mathrm{T}_{\mathrm{f}_{\mathrm{I}}(\mathrm{e})}(t) * \mathrm{~T}_{\mathrm{f}_{\mathrm{I}}(\mathrm{e})}(-t)=\mathrm{T}_{\mathrm{f}_{\mathrm{I}}(\mathrm{e})}(t) * \mathrm{~T}_{\mathrm{f}_{\mathrm{I}}(\mathrm{e})}(t)=\mathrm{T}_{\mathrm{f}_{\mathrm{I}}(\mathrm{e})}(t)$

$\mathrm{F}_{\mathrm{f}_{\mathrm{I}}(\mathrm{e})}(\theta)=\mathrm{F}_{\mathrm{f}_{\mathrm{I}}(\mathrm{e})}(t+(-t)) \leq$

$\mathrm{F}_{\mathrm{f}_{\mathrm{I}}(\mathrm{e})}(t) \Delta \mathrm{F}_{\mathrm{f}_{\mathrm{I}}(\mathrm{e})}(-t)=\mathrm{F}_{\mathrm{f}_{\mathrm{I}}(\mathrm{e})}(t) \Delta \mathrm{F}_{\mathrm{f}_{\mathrm{I}}(\mathrm{e})}(t)=\mathrm{F}_{\mathrm{f}_{\mathrm{I}}(\mathrm{e})}(t)$

Hence proved.

\section{Proposition 3.6:}

An IFSS I on $(\mathrm{V}, \mathrm{K})$ is said to be an IFSLS with respect to the set $\mathrm{E}$ if and only if the following equations hold

$$
\begin{aligned}
\left.T_{f_{I}(e)}(\lambda s+\mu t)\right) \geq & T_{f_{I}(e)}(s) * T_{f_{I}(e)}(t) \\
\left.F_{f_{I}(e)}(\lambda s+\mu t)\right) & \leq F_{f_{I}(e)}(s) \Delta F_{f_{I}(e)}(t) \forall s, t \in V, \lambda, \mu \\
& \in F, e \in E
\end{aligned}
$$

If $a * b=\min \{a, b\}, a \Delta b=\max \{a, b\}$

Proof: First suppose I be an IFSLS onV(K) w.r.t.E.

Then $\left.\quad T_{f_{I}(e)}(\lambda s+\mu t)\right) \geq T_{f_{I}(e)}(\lambda s) * T_{f_{I}(e)}(\mu t) \geq$

$T_{f_{I}(e)}(8) * T_{f_{I}(e)}(t)$

$\left.F_{f_{I}(e)}(\lambda s+\mu t)\right) \leq F_{f_{I}(e)}(\lambda s) \Delta F_{f_{I}(e)}(\mu t)$

$$
\leq F_{f_{I}(e)}(s) \Delta F_{f_{I}(e)}(t)
$$

Conversely by proposition 3.5 ,

$$
\begin{gathered}
T_{f_{I}(e)}(\lambda s)=T_{f_{I}(e)}(\theta+\lambda s) \geq T_{f_{I}(e)}(\theta) * T_{f_{I}(e)}(s) \\
\geq T_{f_{I}(e)}(s) * T_{f_{I}(e)}(s) \\
=T_{f_{I}(e)}(s) F_{f_{I}(e)}(\lambda s)=F_{f_{I}(e)}(\theta+\lambda s) \\
\leq F_{f_{I}(e)}(\theta) \Delta F_{f_{I}(e)}(s) \\
\leq F_{f_{I}(e)}(s) \Delta F_{f_{I}(e)}(s)=F_{f_{I}(e)}(s) \\
T_{f_{I}(e)}(s+t)=T_{f_{I}(e)}(s+(-1)(-t)) \\
\geq T_{f_{I}(e)}(s) * T_{f_{I}(e)}(-t) \\
\geq T_{f_{I}(e)}(s) * T_{f_{I}(e)}(t)
\end{gathered}
$$

$$
\begin{aligned}
& F_{f_{I}(e)}(s+t)=F_{f_{I}(e)}(s+(-1)(-t)) \\
& \leq F_{f_{I}(e)}(s) \Delta F_{f_{I}(e)}(-t) \leq F_{f_{I}(e)}(s) \Delta F_{f_{I}(e)}(t)
\end{aligned}
$$

Hence the proof.

Theorem 3.7: Let $I_{1}$ and $I_{2}$ be two IFSLSs over (V (K), E).Then $I_{1} \cap I_{2}$ is also an $\operatorname{IFSLS}$ over $(\mathrm{V}(\mathrm{K}), \mathrm{E})$.

Proof: Let $I_{1} \cap I_{2}=P$.Now for $s, t \in V$

$T_{f_{p}(e)}(s+t)=T_{f_{I_{1}}(e)}(s+t) * T_{f_{I_{2}}(e)}(s+t) \geq$

$\left[T_{f_{I_{1}}(e)}(s) * T_{f_{I_{1}}(e)}(t)\right] *\left[T_{f_{I_{2}}(e)}(s) * T_{f_{I_{2}}(e)}(t)\right]$

$=\left[T_{f_{I_{1}}(e)}(s) * T_{f_{I_{2}}(e)}(t)\right] *\left[T_{f_{I_{1}}(e)}(t) * T_{f_{I_{2}}(e)}(s)\right]$

$=T_{f_{I_{1}}(e)}(s) *\left[T_{f_{I_{1}}(e)}(t)\right] *\left[T_{f_{I_{2}}(e)}(t)\right] * T_{f_{I_{2}}(e)}(s)$

$=T_{f_{I_{1}}(e)}(s) * T_{f_{P}(e)}(t) * T_{f_{I_{2}}(e)}(s)$

$=T_{f_{I_{1}}(e)}(s) * T_{f_{I_{2}}(e)}(s) * T_{f_{P}(e)}(t)($ as $*$ is commutative $)$

$=T_{f_{P}(e)}(s) * T_{f_{P}(e)}(t)$

Hence $T_{f_{p}(e)}(s+t) \geq T_{f_{P}(e)}(s) * T_{f_{P}(e)}(t)$

Also, $\quad T_{f_{P}(e)}(\lambda s)=T_{f_{I_{1}}(e)}(\lambda s) * T_{f_{I_{2}}(e)}(\lambda s) \geq T_{f_{I_{1}}(e)}(s) *$

$T_{f_{I_{2}}(e)}(s)=T_{f_{P}(e)}(s)$

Thus, $T_{f_{P}(e)}(\lambda s) \geq T_{f_{P}(e)}(s)$ for $\lambda \in K$.

Similarly $\quad F_{f_{p}(e)}(s+t) \leq F_{f_{P}(e)}(s) \Delta F_{f_{P}(e)}(t) \quad$ and $F_{f_{P}(e)}(\lambda s) \geq F_{f_{P}(e)}(t)$ for $\lambda \in K$.

Hence proved.

Remark 3.8: For two IFSLS $I_{1}$ and $I_{2} \operatorname{over}(\mathrm{V}(\mathrm{K}), \mathrm{E})$, $I_{1} \cup I_{2}$ is not generally an $\operatorname{IFSLS}$ over $(\mathrm{V}(\mathrm{K}), \mathrm{E})$.It is possible if anyone is contained in another. For instance, let us consider two IFSLS s $I_{1}$ and $I_{2}$ over the real vector space $\mathrm{V}=R^{2}$ and the parametric set $\mathrm{E}=\left[\mathrm{e}_{\mathrm{i}} / \mathrm{i}=1,2\right\}$ as following:

$T_{f_{I_{1}}\left(e_{i}\right)}(x)=\left\{\begin{array}{c}\frac{1}{2} \text { ifithcoordinateof } x \in R^{2} \text { isnonzeroonly } \\ 0 \text { otherwise }\end{array}\right.$ $F_{f_{I_{1}}\left(e_{i}\right)}(x)=\left\{\begin{array}{c}\frac{2}{5} \text { ifithcoordinateof } x \in R^{3} \text { isnonzeroonly } \\ 1 \text { otherwise }\end{array}\right.$ $T_{f_{I_{2}}\left(e_{i}\right)}(x)=\left\{\begin{array}{c}\frac{2}{5} \text { if ithcoordinateof } x \in R^{2} \text { isnonzeroonly } \\ 1 / 10 \text { otherwise }\end{array}\right.$ $F_{f_{I_{2}}\left(e_{i}\right)}(x)=\left\{\begin{array}{c}0 \text { ifithcoordinateof } x \in R^{2} \text { isnonzeroonly } \\ 1 / 5 \text { otherwise }\end{array}\right.$ If $\mathrm{a} * b=\min \{a, b\}, \mathrm{a} \Delta b=\max \{a, b\}$.

Let $I_{1} \cup I_{2}=P$

$T_{f_{P}\left(e_{1}\right)}(x+y)=T_{f_{I_{1}}\left(e_{1}\right)}(1,1)=\max \left\{0, \frac{1}{10}\right\}=\frac{1}{10}$

$T_{f_{P}\left(e_{1}\right)}(x) *$

$T_{f_{P}\left(e_{1}\right)}(y)=\left\{T_{f_{I_{1}}\left(e_{1}\right)}(x) \Delta T_{f_{I_{1}}(e)}(x)\right\} *\left\{T_{f_{I_{1}}\left(e_{1}\right)}(y) \Delta T_{f_{I_{1}}(e)}(y)\right.$

\}

Published By: 


\section{Depiction of Intuitionistic Fuzzy Soft Linear Spaces}

$=\min \{\max \{1 / 2,1 / 10\}\}, \max \{0,2 / 5\}\}=\min \{1 / 2,2 / 5\}=2 / 5$

Hence $T_{f_{P}\left(e_{1}\right)}(x+y)<T_{f_{P}\left(e_{1}\right)}(x) * T_{f_{P}\left(e_{1}\right)}(y)$ i.e $I_{1} \cup I_{2}$ is not an IFSLS here.

Now if define I over $\left(R^{3}, E\right)$ as following

$T_{f_{I_{1}}\left(e_{1}\right)}(x)=\left\{\begin{array}{c}\frac{1}{6} \text { ifithcoordianateof } x \in R^{2} \text { isnonzeroonly } \\ 0 \text { otherwise }\end{array}\right.$ $F_{f_{I_{1}}\left(e_{1}\right)}(x)=\left\{\begin{array}{c}\frac{7}{10} \text { if ithcoordianateof } x \in R^{2} \text { isnonzeroonly } \\ 1 \text { otherwise }\end{array}\right.$

It can be easily verified that $I_{2} \subseteq I_{1}$ and $I_{1} \cup I_{2}$ is an IFSLS over $\left(\left(R^{2}(R), E\right)\right.$.

Theorem 3.9: Let $I_{1}$ and $I_{2}$ be two IFSLSs over (V (K),

E).Then $I_{1} \wedge I_{2}$ is also an IFSLS over $(\mathrm{V}(\mathrm{K}), \mathrm{E})$.

Proof: Let $I_{1} \wedge I_{2}=Q$. Now for $\mathrm{x}, \mathrm{y} \in V$ and $(\mathrm{s}, \mathrm{t}) \in E X E$

$T_{f_{Q}(s, t)}(x+y)=T_{f_{I_{1}}(s)}(x+y) * T_{f_{I_{2}}(t)}(x+y) \geq$

$\left[T_{f_{I_{1}}(s)}(x) * T_{f_{I_{1}}(s)}(y)\right] *\left[T_{f_{I_{2}}(t)}(x) * T_{f_{I_{2}}(t)}(y)\right]$

$=\left[T_{f_{I_{1}}(s)}(x) * T_{f_{I_{1}}(s)}(y)\right] *\left[T_{f_{I_{2}}(t)}(y) * T_{f_{I_{2}}(t)}(x)\right]($ as $*$ is

commutative)

$=T_{f_{I_{1}}(s)}(x) *\left[T_{f_{I_{1}}(s)}(y)\right] *\left[T_{f_{I_{2}}(t)}(y)\right] * T_{f_{I_{2}}(t)}(x)($ as $*$ is

associative)

$=T_{f_{I_{1}}(s)}(x) * T_{f_{Q}(s, t)}(y) * T_{f_{I_{2}}(s, t)}(x)$

$=T_{f_{I_{1}}(s)}(x) * T_{f_{I_{2}}(t)}(x) * T_{f_{Q}(s, t)}(y)(]($ as $*$ is commutative $)$

$=T_{f_{Q}(s, t)}(x) * T_{f_{Q}(s, t)}(y)$

Hence $T_{f_{q}(s, t)}(x+y) \geq T_{f_{Q}(s, t)}(x) * T_{f_{Q}(s, t)}(y)$

Also, $T_{f_{Q}(s, t)}(\lambda x)=T_{f_{I_{1}}(s)}(\lambda x) * T_{f_{I_{2}}(t)}(\lambda x) \geq T_{f_{I_{1}}(s)}(x) *$

$T_{f_{I_{2}}(t)}(x)=T_{f_{Q}(s, t)}(x)$

Thus, $T_{f_{Q}(s, t)}(\lambda x) \geq T_{f_{Q}(s, t)}(x)$ for $\lambda \in K$.

Similarly $\quad F_{f_{Q}(s, t)}(x+y) \leq F_{f_{Q}(s, t)}(x) \Delta F_{f_{Q}(s, t)}(y) \quad$ and $F_{f_{Q}(s, t)}(\lambda x) \leq F_{f_{Q}(s, t)}(x)$ for $\lambda \in K$.

Hence proved.

\section{CARTESIAN PRODUCT OF INTUITIONISTIC FUZZY SOFT LINEAR SPACES}

Definition4.1: Let $I_{1}$ and $I_{2}$ be two IFSLSs over (V (K), E) and $(\mathrm{W}(\mathrm{K}), \mathrm{E})$ respectively. Then their Cartesian product is $I_{1} X I_{2}=C$ where $f_{C}(s, t)=f_{I_{1}}(s) X f_{I_{2}}(t)$ for $(\mathrm{s}, \mathrm{t}) \in E X E$. Analytically

$$
\begin{gathered}
f_{C}(s, t)=\left\{<(x, y), T_{f_{C}(s, t)}(x, y), F_{f_{C}(s, t)}(y)>/(x, y)\right. \\
\in V X W\}
\end{gathered}
$$

With $T_{f_{C}(s, t)}(x, y)=T_{f_{I_{1}}(s)}(x) * T_{f_{I_{2}}(t)}(y)$

$F_{f_{C}(s, t)}(x, y)=F_{f_{I_{1}}(s)}(x) \Delta F_{f_{I_{2}}(t)}(y)$

This can be extended for more than two IFSLS s.
Theorem 4.2: Let $I_{1}$ and $I_{2}$ be two IFSLSs over (V (K), E) and $(\mathrm{W}(\mathrm{K}), \mathrm{E})$ respectively. Then their Cartesian product $I_{1} X I_{2}$ is an IFSLS over ([VXW](k),EXE).

Proof: Let $I_{1} X I_{2}=C$ where $f_{C}(s, t)=f_{I_{1}}(s) X f_{I_{2}}(t)$ for $(\mathrm{s}$, t) $\in E X E$

Then for $\left(x_{1}, y_{1}\right),\left(x_{2}, y_{2}\right) \in V X W$

$T_{f_{C}(s, t)}\left[\left(x_{1}, y_{1}\right)+\left(x_{2}, y_{2}\right)\right]=T_{f_{C}(s, t)}\left[\left(x_{1}+x_{2}, y_{1}+\right.\right.$ $\left.\left.y_{2}\right)\right]=T_{f_{I_{1}}(s)}\left(x_{1}+x_{2}\right) * T_{f_{I_{2}}(t)}\left(y_{1}+y_{2}\right)$

$\geq\left[T_{f_{I_{1}}(s)}\left(x_{1}\right) * T_{f_{I_{1}}(s)}\left(x_{2}\right)\right] *\left[T_{f_{I_{2}}(t)}\left(y_{1}\right) * T_{f_{I_{2}}(t)}\left(y_{2}\right)\right]$

$=\left[T_{f_{I_{1}}(s)}\left(x_{1}\right) * T_{f_{I_{2}}(t)}\left(y_{1}\right)\right] *\left[T_{f_{I_{1}}(s)}\left(x_{2}\right) *\right.$

$\left.T_{f_{I_{2}}(t)}\left(y_{2}\right)\right]=T_{f_{C}}(s, t)\left(x_{1}, y_{1}\right) * T_{f_{C}}(s, t)\left(x_{2}, y_{2}\right)$

Similarly

$F_{f_{C}}(s, t)\left[\left(x_{1}, y_{1}\right)+\left(x_{2}, y_{2}\right)\right] \leq$

$F_{f_{C}}(s, t)\left(x_{1}, y_{1}\right) \Delta F_{f_{C}}(s, t)\left(x_{2}, y_{2}\right)$

$\operatorname{Next}, T_{f_{C}}(s, t)\left[\lambda\left(x_{1}, y_{1}\right)\right]=T_{f_{C}}(s, t)\left[\left(\lambda x_{1}, \lambda y_{1}\right)\right]=$

$T_{f_{I_{1}}(s)}\left(\lambda x_{1}\right) * T_{f_{I_{2}}(t)}\left(\lambda y_{1}\right) \geq$

$T_{f_{I_{1}}(s)}\left(x_{1}\right) * T_{f_{I_{2}}(t)}\left(y_{1}\right)=T_{f_{s}}(s, t)\left(x_{1}, y_{1}\right)$

Similarly $F_{f_{s}}(s, t)\left[\lambda\left(x_{1}, y_{1}\right)\right] \leq F_{f_{s}}(s, t)\left(x_{1}, y_{1}\right)$

\section{EXPERIMENTS AND RESULTS DESCRIPTION}

Definition 5.1: Let $I_{1}$ and $I_{2}$ be two IFSLS over (V (K), E).Then $I_{1}$ is IFSS of $I_{2}$ if $I_{1} \subseteq I_{2}$ i.e. $T_{f_{I_{1}}(e)}(u) \leq$ $T_{f_{I_{2}}(e)}(u)$

$F_{f_{I_{1}}(e)}(u) \geq F_{f_{I_{2}}(e)}(u) \forall u \in V, e \in E$

Example 5.2: Let us consider two IFSLS s $I_{1}$ and $I_{2}$ over real vector space $\mathrm{V}=\mathrm{R}^{3}$ and parametric set $\mathrm{E}=\{\mathrm{e}\}$ as following:

$$
\begin{aligned}
& T_{f_{I_{1}}(e)}(x)=\left\{\begin{array}{l}
\frac{1}{4} \text { if } x \in(a, b, c) \in R^{3}, a+b+c=0 \\
0, \text { otherwise }
\end{array}\right. \\
& F_{f_{I_{1}}(e)}(x)=\left\{\begin{array}{r}
0, \text { if } x \in(a, b, c) \in R^{3}, a+b+c=0 \\
1 / 6, \text { otherwise }
\end{array}\right. \\
& T_{f_{I_{2}}(e)}(x)=\left\{\begin{array}{c}
\frac{1}{2} \text { if } x \in(a, b, c) \in R^{3}, a+b+c=0 \\
2 / 7, \text { otherwise }
\end{array}\right.
\end{aligned}
$$

$F_{f_{I_{2}}(e)}(x)=\left\{\begin{array}{c}0, \text { if } x \in(a, b, c) \in R^{3}, a+b+c=0 \\ 1 / 9, \text { otherwise }\end{array}\right.$

If $\quad \mathrm{a} * b=\max \{a+b-1,0\} \quad$ and $\quad \mathrm{a} \Delta b=\min \{a+$ $b, 1\}$.Then $I_{1}$ is an intuitionistic fuzzy soft subspace of $I_{2}$ over $\left(R^{3}(R), E\right)$. 
Corollary 5.3:Let $I$ be an IFSLS over (V(K),E).Then for arbitrary but fixed $\lambda \in K, \lambda I=\left\{e, \frac{\lambda f_{I}(e)}{e} \in E\right\}$ is also a IFSLs over $(\mathrm{V}(\mathrm{K}), \mathrm{E}) \quad$ where $\lambda f_{I}(e)=\left\{\left\langle\lambda x, T_{f_{I}(e)}(x), F_{f_{I}(e)}(x)\right\rangle\right.$ $/ x \in V\}$.Moreover $\lambda I$ is a intuitionistic fuzzy soft subspace of I.

\section{Proof: Clearly $\lambda x \in V$ for $x \in V, \lambda \in K$}

Since I be an IFSLS over (V (K), E) so by construction of $\lambda I$

$T_{f_{I}(e)}(\lambda x+\lambda y) \geq T_{f_{I}(e)}(\lambda x) * T_{f_{I}(e)}(\lambda y)$

$F_{f_{I}(e)}(\lambda x+\lambda y) \leq F_{f_{I}(e)}(\lambda x) * F_{f_{I}(e)}(\lambda y) \forall \lambda x, \lambda y \in V, \mathrm{e} \in E$

$T_{f_{I}(e)}(\mu(\lambda x)) \geq T_{f_{I}(e)}(\lambda x)$

$F_{f_{I}(e)}(\mu(\lambda x)) \leq F_{f_{I}(e)}(\lambda x) \forall \lambda x \in V, \mathrm{e} \in E, \mu \in K$

Hence $\lambda I$ is IFSLS over $(\mathrm{V}(\mathrm{K}), \mathrm{E})$

$\operatorname{Next} T_{f_{I}(e)}(x)=T_{f_{I}(e)}\left(\lambda^{-1}(\lambda x)\right) \geq T_{f_{I}(e)}(\lambda x)$

$F_{f_{I}(e)}(x)=F_{f_{I}(e)}\left(\lambda^{-1}(\lambda x)\right) \geq F_{f_{I}(e)}(\lambda x)(\forall \lambda \neq 0) \in$

$K, x \in V, \mathrm{e} \in E$

Then $\lambda$ IisIFS subspace of I.

Corollary 5.4: Let $I_{1}$ be an IFSLS over (V (K), E).Then for arbitrary but fixed $\lambda, \mu \in K . \lambda I_{1}+\mu I_{1}=\left\{\mathrm{e},\left(\lambda f_{I_{1}}+\mu f_{I_{1}}\right)(e) /\right.$ $\mathrm{e} \in E\}$ is again in IFSLS over $(\mathrm{V}(\mathrm{K}), \mathrm{E})$ where , $\left(\lambda f_{I_{1}}+\right.$ $\left.\mu f_{I_{1}}\right)(e)=\left\{\left\langle(\lambda x+\mu y), T_{f_{I_{2}}(e)}(\lambda x+\mu y), F_{f_{I_{2}}(e)}(\lambda x+\right.\right.$ $\mu y>/ \mathrm{x}, \mathrm{y} \in V\}$

Moreover $\lambda I_{1}+\mu I_{1}$ is an IF soft subspace of $I_{1}$

Proof: Since V $(\mathrm{K})$ is a vector space.

So $\mathrm{x}+\mathrm{y}, \lambda x+\mu y \in V$ for $\mathrm{x}, \mathrm{y} \in V$ and $\lambda, \mu \in F$.

Hence the proof is completed.

Corollary 5.5: Let $f_{I_{1}}(e), e \in E$ be a IF subspace on V $(\mathrm{K})$ where $I_{1}$ is an IFSLS over $(\mathrm{V}(\mathrm{K}), \mathrm{E})$.Then $\lambda f_{I_{1}}(e)=$ $\left\{<\lambda x, T_{f_{I_{1}}(e)}(\lambda x), F_{f_{I_{1}}(e)}(\lambda x)>/ x \in V\right\}$

Proof: It is obvious

For instance, if $\mathrm{V}=\{\mathrm{x}, \mathrm{y}, \mathrm{z}\}$ and $\mathrm{k}=\{\lambda, \mu\}$ then $\lambda x, \lambda y, \lambda z, \mu x, \mu y, \mu z \in V$ and $\lambda x+\lambda x, \lambda x+\lambda y, \lambda x+\mu x, \mu y+$ $\mu y \ldots \ldots . . . \in \mathrm{V}$.

Since $f_{I_{1}}(e)$, e $\in E$ is an IFS subspace on V $(\mathrm{K})$ so all the inequalities hold good.

\section{REFERENCES}

1. A.sezgin sezer, A.O.Atagin, A new kind of vector space: soft vector space, southest asian Bulletin of Mathematics(2016040:753-770.

2. D. Molodtsov, Soft set theory-First results, Computers Math. Applic. 37 (4/5), 19-31, (1999).

3. K. Atanassov and S. Stoeva, Intuitionistic fuzzy sets, in: Polish Symp.

4. K. Atanassov, Intuitionistic fuzzy sets, in: V. Sgurev, Ed., VII ITKR's Session, Sofia, June 1983 (Central Sci. and Techn. Library, Bulg. Academy of Sciences, 1984).
5. Moumita Chiney,S.K.Samanta,Intuitionistic fuzzy dimension of an intuitionistic fuzzy vector space,Notes onintuitionistic fuzzy sets,vol 24,2018, No 1,21-29.

6. P.K. Maji, R. Biswas, A.R. Roy, Soft set theory, Comput. Math. Appl 45 (2003) 555-562. 